\title{
TBM
}

\section{Design and implementation of decision support for tobacco dependence treatment in an inpatient electronic medical record: a randomized trial}

\author{
Steven L. Bernstein, MD, ${ }^{1}$ June Rosner, MA, MEd, ${ }^{2}$ Michelle DeWitt, RN, ${ }^{3}$ Jeanette Tetrault, MD, ${ }^{4}$ \\ Allen L. Hsiao, MD, ${ }^{5}$ James Dziura, PhD, ${ }^{2}$ Scott Sussman, MD, ${ }^{3}$ Patrick O'Connor, MD, MPH, ${ }^{4}$ Benjamin Toll ${ }^{6}$
}

Emerg. Med., Yale School Med. Health Policy, Public Health, 464 Congress Ave., Suite 260, New Haven, CT 06519, USA

${ }^{2}$ Emerg. Med., Yale School Med., New Haven, CT, USA

${ }^{3}$ Hospital, New Haven, CT, USA

${ }^{4}$ Internal Med., Yale School Med., New Haven, CT, USA

${ }^{5}$ Pediatrics, Emerg. Med., Hospital, New Haven, CT, USA

${ }^{6}$ Psychiatry, Yale School Med., Pop. Health Sciences/MUSC, New Haven, CT, USA

Correspondence to: $\mathrm{S}$ Bernstein steven.bernstein@yale.edu

Cite this as: TBM 2017;7:185-195 doi: 10.1007/s13142-017-0470-8

(c) Society of Behavioral Medicine 2017

\section{Abstract}

Tobacco dependence treatment for hospitalized smokers results in long-term cessation if treatment continues at least 30 days post-discharge. Health information technology may facilitate ongoing tobacco dependence treatment after hospital discharge. To describe the use and impact of a new decision support tool and order set for inpatient physicians, addressing tobacco dependence treatment for hospitalized smokers, embedded in an electronic health record (EHR). In a cluster-randomized trial, 254 physicians were randomized (1:1) to either receive or not receive the decision support tool and order set, which were embedded in the Epic (Madison, WI) EHR used at 2 hospitals in a single city. When an adult patient was admitted to a medical service, an electronic alert appeared if the patient was coded in the EHR as a smoker. For physicians randomized to the intervention, the alert linked to an order set to prescribe tobacco treatment medications and refer the patient to the state tobacco quitline. Additionally, "tobacco use disorder" was added to the patient's problem list, and an e-mail was sent to the patient's primary care provider (PCP). In the control arm, an alert fired with no screen visibility. Generalized estimating equations were used to model the data. Since August 2013, the alert has appeared for 10,939 patients (5391 intervention, 5548 control). Compared to control physicians, intervention physicians were more likely to order tobacco treatment medication (35 vs. $29 \%$, $P<0.0001)$, populate the problem list with tobacco use disorder ( 41 vs. $2 \%, P<0.0001$ ), and make a referral to the state smokers' quitline (30 vs. $0 \%, P<0.0001$ ). In addition, intervention physicians sent an e-mail to the patient's PCP 4152 (99\%) times. Designing and implementing an order set and alert for tobacco treatment in an EHR is feasible and helps physicians place more orders for tobacco treatment medication, referrals to the state smokers' quitline, and e-mails to patients' PCPs. Data on cessation outcomes are pending. Trial registration: www. ClinicalTrials.gov (NCT01691105).

\section{Keywords}

Smoking cessation, Tobacco dependence treatment, Decision support, Electronic health records

\section{Implications}

Practice: Enhancing the treatment of hospitalized smokers with electronic decision support is feasible, and can lead to dramatic improvements in processes of care.

Policy: Because of the near-universality of electronic health records and telephone quitlines in developed countries, and the extensive literature demonstrating the clinical efficacy and cost effectiveness of tobacco dependence treatment, electronic decision support for tobacco is a scalable, cost-effective approach to the population-based management of the leading cause of death in the developed world.

Research: Future work should examine the impact of electronic decision support on quit rates, the incidence of subsequent tobacco-related health events, and how to electronically integrate tobacco dependence treatment across all inpatient and outpatient clinical encounters.

\section{Introduction}

Because tobacco dependence remains the leading cause of death and illness in the USA, smoking cessation and tobacco dependence treatment has long been a publicly reported standard of the quality of inpatient care. Screening and treatment for tobacco use is part of the core measure set used by the Center for Medicare and Medicaid Services (CMS) for patients admitted with acute myocardial infarction, pneumonia, or congestive heart failure. It is a core measure of the National Quality Forum and one of the choices in the optional measure set offered by the Joint Commission [1]. 
Hospital-based providers' ability to identify and treat smokers has been the focus of publicly reported core measures developed and endorsed by the Centers for Medicare and Medicaid Services, the Joint Commission, and the National Quality Forum. Hence, the recording of tobacco use was identified as an early indicator of meaningful use of electronic health records by the Health Information Technology for Economic and Clinical Health Act (HITECH). [2].

Healthcare systems increasingly look to health information technology (HIT) to improve the quality and efficiency of care, avoid unnecessary testing, and meet federally mandated targets for meaningful use. The impact of electronic alerts and notifications on the quality of care remains unclear. While some studies have shown improvements in the processes of care, including adherence to practice guidelines, the impact of HIT on the quality of care and clinical outcomes remains unclear $([3,4])$.

To facilitate the identification and treatment of individuals with tobacco dependence, healthcare systems are increasingly looking to EHRs. Besides the simple capture of tobacco use status, EHRs can offer additional functionality to help clinicians treat smokers. Examples include electronic prompts to offer medication, automated referrals to a tobacco dependence treatment service, and electronic referral to a tobacco quitline. For example, in the USA veterans hospital system, the Tobacco Tactics program, consisting of physician and nurse training, written materials, and an electronic reminder for physicians, has been shown to increase provision of counseling and medications to hospitalized smokers [5]. Data on longterm quit rates were not reported.

There is a paucity of research published to date assessing the efficacy of EHRs in promoting providers' treatment of tobacco dependence, although studies addressing the issue have suggested positive outcomes. One study of a closed-loop, bidirectional electronic referral to the state tobacco quitline, conducted in two primary care clinics in a single healthcare system, found that the proportion of smokers referred increased from 0.3 to $14 \%$ after the e-referral was implemented [6]. Changes in clinical outcomes, such as tobacco abstinence, were not reported.

A common challenge in the treatment of tobacco dependence among hospitalized smokers is that relapse to smoking is common after discharge. Comprehensive programs that extend tobacco dependence treatment at least 30 days after hospital discharge via counseling, medication treatment, or both have been shown to result in a higher proportion of sustained quits [7]. Therefore, a specific goal of this project was to develop and test a set of tools, embedded in the EHR, which could facilitate the continued treatment of tobacco dependence with evidence-based interventions after smokers were discharged from a hospitalization. We were interested both in the ability of these electronic tools to improve providers' treatment of hospitalized smokers, and whether this enhanced treatment might improve quit rates among smokers after hospital discharge.
To our knowledge, there have been no reports assessing the ability of a more comprehensive set of EHR tools to improve the sustained treatment of tobacco dependence, or improve cessation rates among smokers treated by providers using those tools. The goal of this paper is to describe the design and implementation of such a system and to provide evidence regarding its efficacy in changing physician practice. Clinical outcomes are still being assessed and will be the subject of future reports.

\section{Methods}

The study was approved by the institution's Human Investigation Committee and registered at www. ClinicalTrials.gov (NCT01691105).

This was a two-arm prospective clinical trial, with two groups of subjects-physicians and patients. Randomization occurred at the level of the physician. The primary endpoint was biochemically verified 1-year tobacco cessation among the patients, performed via in-person carbon monoxide breath testing 1 year after enrollment, for subjects self-reporting by phone tobacco abstinence. The most important secondary endpoint was the provision of tobacco dependence treatment by the physicians. Using Curran's taxonomy for study designs assessing both implementation and clinical effectiveness, this was a Type I design: effectiveness was the primary endpoint, but data assessing important implementation measures are collected and reported [8].

Development of the E-STOPS-The Information Technology Services (ITS) team developed within the Epic EHR a best practice alert, the vendor's version of medico-logic modules for decision support. The alert set-up consisted of a base record and six criteria records, with the base record holding those criteria records and the data displayed to the provider.

The first criterion incorporated a rule attached to the patient's history to see if smoking status was flagged as current. To capture the physicians enrolled in the study, criterion two created a group record of all the providers who were part of the E-STOPS arm. A similar group record was created for control physicians. If the control portion of the alert appeared, flagging the patient as being cared for by a control provider, then criterion three instructed the intervention E-STOPS portion of the alert not to appear.

The alert was set to appear only for the first $24 \mathrm{~h}$ of the patient's stay, when admission orders were written for patients. The alert also was set not to appear in certain situations where a patient may not be stable enough to answer smoking status, such as in the emergency department (ED). These exceptions were controlled by the fourth and fifth criteria. The last (sixth) criterion behind the alert flagged patients as being part of the study the first time they were admitted. This ensured that the alert would not appear again on subsequent admissions or hospital encounters. This kept control patients as part of the control group and intervention 


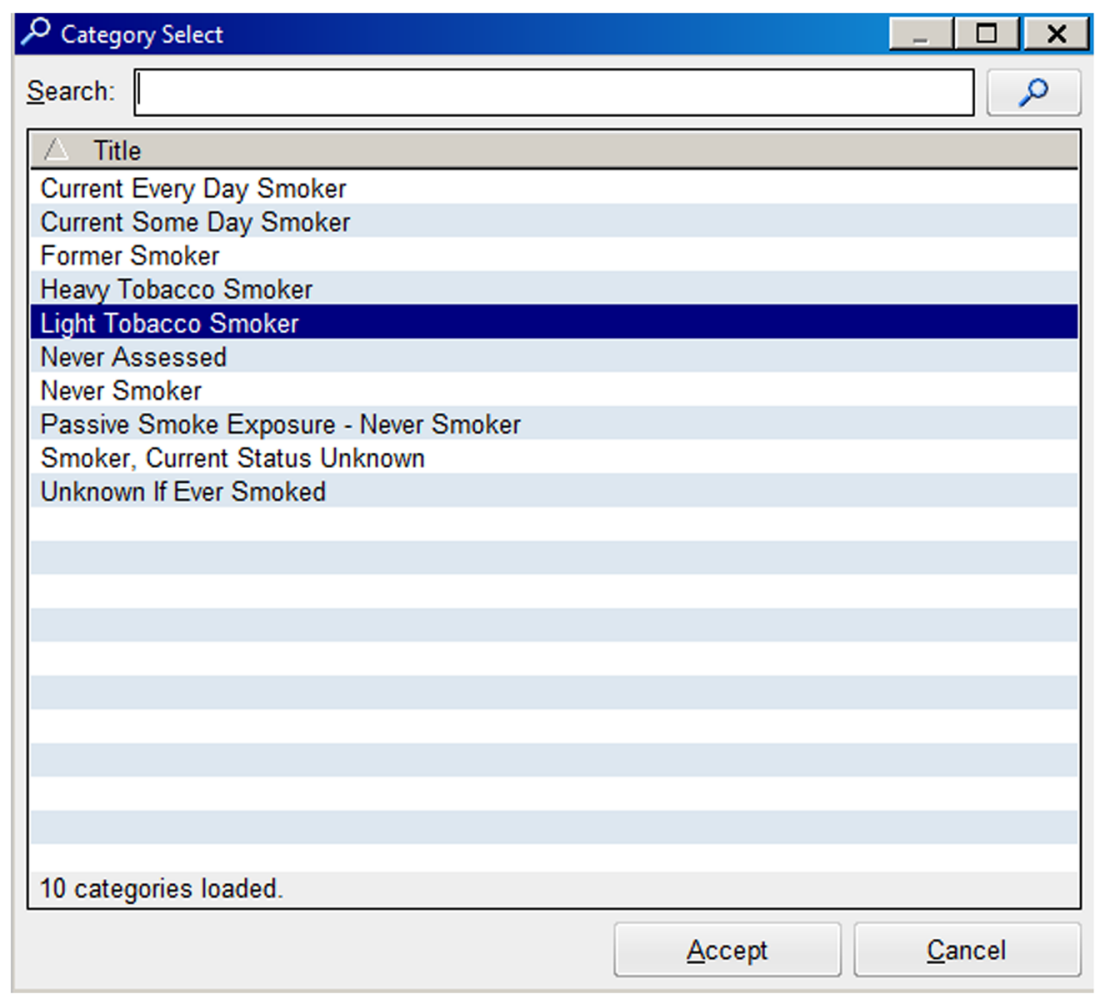

Fig. 1 Smoking status options in electronic health record

patients as part of the intervention group if they were readmitted.

The components of the E-STOPS are shown in Figs. 1 and 2, and online Figs. 3, 4, 5, 6, and 7. Figure 1 depicts the responses in the electronic record available to the provider to record smoking status. The responses which designated the patient as a smoker, and which subsequently caused the alert to appear, are "Current every day smoker," "Current some day smoker," "Heavy smoker," "Light smoker," and "Smoker, current status unknown."

Figure 2 depicts the best practice alert, or alert. The alert appeared visibly if the physician logged on in the E-STOPS arm; it appeared silently if the physician was in the control arm (in other words, the alert records if a control physician logs on to the chart of a smoker, but the physician sees nothing on the screen). Of note, the alert has three functions that were pre-checked, for the physician, if s/he accepted the alert: (1) a referral to the Connecticut State Smokers' Quitline, (2) opening of the E-STOPS order set, and (3) adding "tobacco use disorder" to the patient's problem list. This saved clinician time while allowing them the autonomy to not order the interventions if they chose. An additional function, an EMR-embedded e-mail sent to the patient's primary care provider that tobacco dependence treatment was begun, appeared irrespective of whether the physician accepts the alert.

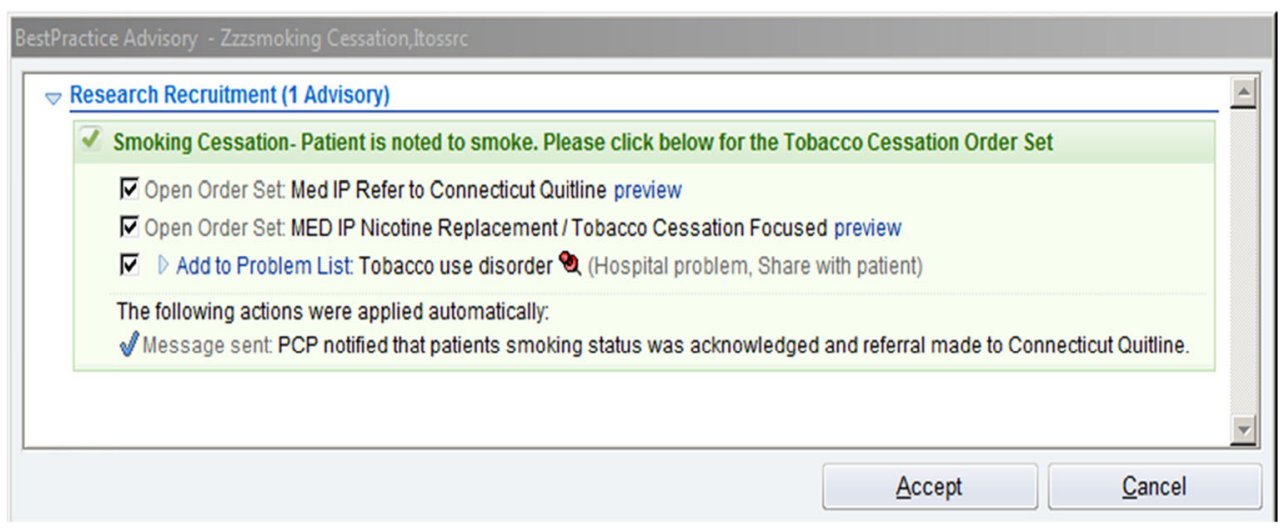


Control arm providers were able to execute these functions individually, if desired. They could fax a paper-based referral to the tobacco quitline; order tobacco treatment medications already on the hospital formulary; add tobacco use disorder to the problem list, just like any diagnosis may be added; and send an e-mail to the primary care provider. These steps each would have entailed additional clicks and text entry.

Figure 3 in the Appendix shows the E-STOPS itself. The order set covered most FDA-approved medications and reminded the physician that an electronic referral was made to the state quitline. All components of E-STOPS were consistent with federal guidelines for the treatment of tobacco dependence. [9] The tabs for "Nursing" and "Ancillary Consults" were not activated, because of resource constraints.

Figure 4 shows the brief EMR-embedded e-mail message sent to the patient's primary care provider. Of note, most providers in our system use Epic. There are a small number of independent medical practices, and one local federally qualified health center, which do not utilize Epic in their practices and either is on paper or on EMRs that cannot receive direct messages. We were unable to send e-mail messages to these providers.

Because these communications were secure, embedded in the EHR, and only between study providers and primary care physicians, HIPAA compliance was maintained.

Figure 5 shows the text that was inserted into the discharge summary of smokers treated by E-STOPS physicians.

Figure 6 displays a feedback report sent every 3 months to E-STOPS physicians. The report compared an individual physicians' performance to the mean of his/her colleagues. Additional details of the report's contents are provided in the "Discussion" section.

Figures 7 displays the alert base record used to create the physician groups. Along with an encoding rule, this allowed us to randomize by provider.

Physician recruitment-Between August 2013 and July 2014, physicians were recruited during regularly scheduled conference sessions and staff meetings. Most providers were internal medicine or emergency medicine interns, recruited during their June orientation sessions. A 40-min lecture about the health risks and treatment of tobacco dependence was presented by a study investigator. Written informed consent was given by physicians. Consent forms stated that investigators were studying the effect of an electronic order set on the treatment of hospitalized smokers, and that half the physicians would be randomized to that order set. The form noted the study was voluntary, all reported data would be deidentified, and that physicians could decline to participate without any professional consequences. Randomization was performed on-site by a research assistant using a random number generator (www.randomization.com) to generate the randomization scheme, with 1:1 randomization and stratification by specialty. Physicians randomized to the control arm were asked to leave the room, and the intervention physicians were given an additional 5-10-min lecture describing the functionality and use of the Electronic Support Tool and Orders for the Prevention of Smoking (E-STOPS). Thus, in this realworld effectiveness trial, group assignment was not blinded, but allocation was concealed, and outcome assessments were blinded.

Additional residents were recruited in a similar fashion during didactic sessions held at a hospital-based primary clinic. Hospitalists were recruited during regular monthly staff meetings. To accommodate hospitalists' schedules, trainings were briefer, typically $10-15 \mathrm{~min}$ in duration.

Permission to approach house officers and hospitalists was given by the directors of the residents in traditional medicine, primary care medicine, medicine-pediatrics, emergency medicine, as well as the director of the hospitalist service. All physicians gave written, informed consent. To minimize assessment burden, we did not capture demographic data such as age, gender, race, or ethnicity (other than year of training and specialty) for the physicians in the study.

Patient recruitment-Patients were recruited 7 days/ week by research assistants (RAs). The primary purpose of recruiting patients was to assess and report clinical outcomes. Potential subjects were identified by means of a report generated daily by the clinical information system, which provided the research team with a list of all smokers admitted to the adult medicine services, and their room locations. RAs would go to patients' rooms, introduce themselves, reaffirm smoking status, and explain the goals of the study. Patient subjects then provided written informed consent.

Patients were told that they were part of a study to evaluate their tobacco use after hospital discharge, and that they were consenting to a baseline assessment, three waves of telephone follow-up, and an in-person test of tobacco use if self-reporting abstinence at 1 year. They were informed that they and their providers could treat their tobacco dependence as they saw fit. Patients initially treated by physicians in the intervention arm were assigned to the intervention, for the index admission and all subsequent admissions. Patients treated by physicians in the control condition were assigned to the control for the index admission and all subsequent admissions.

Operationalizing E-STOPS-During the design of the E-STOPS, we had to make a number of decisions regarding functionality. These included decisions around timing and duration of the prompt, prompt triggers, wherein clinicians should see the prompt, mapping patients to clinicians, order set functionalities, prompt suppression on subsequent admissions, training and audit/feedback mechanisms, and nursing involvement. To assist others who may attempt similar work, we wish to provide some details on these issues.

Timing and duration-A critical question was whether to suppress the alert after a specific duration of hospitalization. The clinical concern was that many smokers who might benefit from the alert would be too acutely 
ill to discuss tobacco dependence treatment at the moment of admission. Moreover, the physician might have other urgent priorities, such as providing critical interventions and treatments, and tobacco dependence treatment would be deferred. Still, we wanted to give the provider an opportunity to reconsider treating tobacco dependence at some later time. Because an overarching goal of this project was to create functionalities that physicians would find helpful, and not intrusive, we elected to have the alert appear only for the first $24 \mathrm{~h}$ of admission. If the provider accepted it at any moment in those $24 \mathrm{~h}$, it was suppressed on subsequent openings of the chart. Additionally, if the provider did not accept it in $24 \mathrm{~h}$, it was suppressed. A related issue concerned the possibility of contamination on readmission. If a patient treated by a control provider on the index admission was later readmitted to an E-STOPS provider, we suppressed the alert on the later admission. Conversely, if a patient treated by an E-STOPS provider was readmitted to another ESTOPS provider, the alert was permitted to appear again. This provided a booster dose of decision support for the physician: control arm subjects remained in control conditions on readmission, and E-STOPS arm subjects remained in E-STOPS conditions on readmissions.

Triggers-There are various responses in our tobacco use status box that we mapped to "current smoking." These are provided in Fig 1, and include "current every day smoker," "current some day smoker," "heavy tobacco smoker," "light tobacco smoker," and "smoker, current status unknown." Some of these choices are potentially redundant, but they exist to ensure compliance with meaningful use rules. Thus, we chose to include them all. If any provider clicks any of these categories at any time prior to the study provider's initial opening the chart during the index admission, the alert appears.

Clinician specialties, roles-We elected to test the ESTOPS on medical services only. For this study, we chose not to implement on the following specialty services: psychiatry, because these patients often require more intensive treatment for tobacco dependence, which may be viewed as of lesser importance than their acute behavioral and mood problems, or other addictions; labor and delivery, because medications are not widely used in pregnant women who smoke; pediatrics, because few adolescents require inpatient admission, and the efficacy of pharmacotherapy and quitlines is less clear; and surgery, because surgeons often have reservations about the use of nicotine replacement therapy and are less familiar with tobacco dependence treatment than internists and primary care providers. As a result, the providers we chose to train and implement E-STOPS with were the hospitalists and internal medicine house staff who staff the general medicine wards. These included medical stepdown, intensive care, and coronary care units. We also trained emergency medicine house staff, because they spend several months on these inpatient services. The emergency department itself was not a study site.
Clinician mapping-One particular challenge that was not evident during study design was how to assign individual physicians to patients. Because of duty hour restrictions on house staff; use of night float teams; the care of individual patients by teams that included interns, residents, and attendings; and frequent changes of shift and service among hospitalists and house staff, it was not straightforward to identify which physician should be identified as the patient's primary provider of inpatient care. As such, a strategic decision was made to designate the first physician to log onto the inpatient chart as the physician of record. This was typically a senior resident or internal medicine physician, or a hospitalist. In our hospitals' workflows, interns are usually not the first physician providers to open the chart.

Functionalities-To enhance physician use, it was important that the alert and order set include all standard, evidence-based treatments for smoking, including pharmacologic and behavioral. Thus, most FDAapproved nicotine replacement medications are available, as well as bupropion. To provide counseling that continues beyond the day of discharge, we included an electronic referral to the state smokers' quitline, which uses principles of motivational interviewing [10]. One specific function that we could not yet build was to make that electronic referral bidirectional. In other words, the quitline did not return an electronic report which was then embedded in the patient's medical record. They continued to send a traditional faxed report, which was then scanned and entered into the chart. New bidirectional functionality that will allow the quitline to embed its reports in the medical record is under development.

We also wanted the alert and order set to provide a "closed feedback loop" to the primary care providers, to inform them of their patients' enrollment, and to encourage them to continue tobacco dependence treatment. This was also part of the rationale for autopopulating the patient problem list with "tobacco use disorder." Without that function, smoking status was added to the problem list relatively infrequently. Also, this allowed for other specialty physicians in the health system that may treat the patient to refer them for tobacco dependence treatment.

Last, we added additional text to the patient's discharge summary about their tobacco treatment.

Prompt suppression-In an effort to minimize the risk of alert fatigue and the likelihood that clinicians would decline the alert, the alert in the intervention arm was suppressed after it had been used, or after $24 \mathrm{~h}$ of clock time since admission had elapsed. Twenty-four hours was chosen, in consultation with the hospitalist team and internal medicine residents, as a duration of time sufficient to stabilize acutely ill, newly admitted patients who might not initially be well enough to engage in a discussion about tobacco dependence treatment. If a patient treated by an intervention physician was subsequently readmitted, the E-STOPS would appear again. For patients treated by control arm physicians, E-STOPS was suppressed on subsequent admissions. 
Training, audit, and feedback-Training of the physicians on use of the E-STOPS was brief. Again, this was done deliberately; it was thought important to present the study and E-STOPS in a way that would not be perceived as burdensome and to respect the demands on their time. Only minimal training was provided to enhance the generalizability of the E-STOPS. Qualitative work currently in progress will assess physicians' perceptions of the utility of the audit and feedback component of the intervention.

Providers in the intervention arm were given feedback on their performance. Every 3 months, an e-mail was sent to intervention physicians informing them of how often they used the various E-STOPS functions (medication ordering, quitline referral, PCP notification, and populating the problem list with tobacco use disorder). Comparative data on how the other physicians performed were also provided. A sample feedback report is provided in Fig 6. The report was created with input from a hospitalist co-investigator. Neither residency directors nor the director of the hospitalist service were informed about individual physicians' performance, in order to allay any physician concerns that there might be punitive or financial consequences for non-compliance.

Role of nursing-Although nurses can play critical roles in the treatment of tobacco dependence [11], the order set was not made available to them, since most workflows involving order sets are managed by physicians, physician assistants, or nurse practitioners, as is the post-hospital care. Nursing leadership on the inpatient medical units did receive inservice training about the study, and smoking cessation brochures were placed on the inpatient wards, for distribution ad libitum by clinical nurses.

\section{Analytic Plan}

Clinical trial data were recorded in FileMaker Pro and were imported into SAS 9.4. Use of E-STOPS components was modeled using logistic regression with generalized estimating equations to accommodate the clustering of patients by physician. Analyses are per patient, rather than per admission, to reflect each patient's exposure to intervention components. In other words, the analysis includes the index admission and any readmissions that a patient may have had during the study period. We did this because we think it is more clinically pertinent to analyze cumulative patient exposure to E-STOPS' functions. No covariates, other than physician, were included in the models. Spearman's correlation coefficient was used to assess relationships between the use of E-STOPS components. All statistical tests were two-sided, with alpha set at 0.05.

\section{Results}

The E-STOPS was implemented in August, 2013. By July, 2015, 254 physicians were enrolled. These included 44 hospitalists, 180 internal medicine residents training in three related programs (traditional, primary care, and medicine-pediatrics), and 30 emergency medicine residents. Characteristics of the physicians are provided in Table 1 . Of all physicians, 126 were randomized to E-STOPS, 128 to control.

Table 2 shows the utilization of the various components of the E-STOPS from study inception through February, 2016.

Each component was provided more often for intervention subjects than for control. We do not have definitive data on the composition and delivery of email messages to primary care providers from control physicians discussing tobacco treatment, but early qualitative work and feedback suggests this happened infrequently, if at all.

To date, the hospitalists have had the greatest exposure to E-STOPS, with a mean of 210 patients/hospitalist, compared to 43 patients/intern and 55 patients/resident. This is because the hospitalists spend most of their professional time on the inpatient units, while interns and residents rotate through a variety of clinical services, including critical care units and elective rotations. The internal medicine physicians in the intervention arm had a mean of 85 patient encounters, compared to 23 for the emergency medicine physicians. The emergency physicians spend most of their training time in the emergency department, with only $4-8$ weeks per year spent on inpatient internal medicine units.

There was substantial variation in use of the E-STOPS, as shown in Table 3. This table presents data on use of ESTOPS analyzed by physician encounters and includes specialty and level of training. The data include all hospitalizations, including the index admission and any

\begin{tabular}{llllll}
\hline $\begin{array}{l}\text { Table } 1 \text { | Profile of physicians enrolled in study } \\
\text { Postgraduate year of training }\end{array}$ & \multicolumn{2}{l}{$\begin{array}{l}\text { Service } \\
\text { Internal medicine }\end{array}$} & Emergency medicine & Total \\
\hline \multicolumn{7}{l}{ Traditional } & Primary care & Medicine-pediatrics & & 74 \\
\hline 1 & 54 & 16 & 4 & 0 & 78 \\
\hline 2 & 51 & 14 & 0 & 8 & 29 \\
\hline 3 & 13 & 7 & 1 & 9 & 29 \\
\hline Attending & 14 & 5 & 1 & 0 & 44 \\
\hline Total & 44 & 0 & 0 & 30 & 254 \\
\hline
\end{tabular}




\begin{tabular}{|c|c|c|c|}
\hline Function & $\begin{array}{l}\text { Intervention } \\
(N=5391 \text { patients })\end{array}$ & $\begin{array}{l}\text { Control } \\
(N=5548 \text { patients })\end{array}$ & $P$ value \\
\hline Medications ordered, $N(\%)$ & $1827(34 \%)$ & $1591(29 \%)$ & $<0.0001$ \\
\hline $\begin{array}{l}\text { Tobacco use disorder added } \\
\text { to problem list, } N(\%)\end{array}$ & $2245(42 \%)$ & $122(2 \%)$ & $<0.0001$ \\
\hline Referral made to Quitline, $N(\%)$ & $1584(29 \%)$ & $0(0 \%)^{a}$ & $<0.0001$ \\
\hline Email sent to primary care provider, $N(\%)$ & $5375(99 \%)$ & $\mathrm{N} / \mathrm{A}$ & $\mathrm{N} / \mathrm{A}$ \\
\hline
\end{tabular}

readmissions that may have occurred during the subsequent 12 months. This analysis examines each appearance of the prompt. Each admission included at least one, but possibly more than one, physician encounter. For example, if the first physician to see the patient was in the E-STOPS arm but declined to accept the prompt, then it would have fired again if a second E-STOPS physician logged on to the patient's chart in the first $24 \mathrm{~h}$ of hospitalization. Hence, the totals in this table exceed those in Table 2. Physician performance was comparable across specialties and level of training, with the exception of quitline referrals, which were more commonly made by internists and hospitalists.

The results may be interpreted as follows. Using the data in the top left cell, if one ranks the 111 internists in the E-STOPS arm by the proportion of each person's admissions who received nicotine replacement therapy (NRT), the median proportion would be 0.15 . In other words, the doctor at the $50 \%$ percentile prescribed NRT for 15\% of his/her admitted smokers.

In general, physicians who used one E-STOPS function were more likely to use others: the Spearman correlation coefficient between nicotine replacement medication prescribing and quitline referral was $(0.35$, $P<0.001)$; between medication prescribing and placing tobacco use disorder in the problem list $(0.29, P<0.001)$; and between quitline referral and problem list updating (0.56, $P<0.001$ ) (because the e-mail message to the primary care provider was sent automatically when ESTOPS fired, no correlation coefficients are offered).

\begin{tabular}{|c|c|c|c|c|}
\hline \multirow[t]{3}{*}{ E-STOPS function } & \multicolumn{3}{|c|}{$\begin{array}{l}\text { Proportion of admission encounters receiving E-STOPS function, } \\
\text { median (IQR) }\end{array}$} & \multirow[t]{3}{*}{$P$ value } \\
\hline & \multicolumn{3}{|l|}{ Specialty } & \\
\hline & $\begin{array}{l}\text { Internal medicine: } \\
111 \text { MDs, } 9509 \\
\text { admissions }\end{array}$ & $\begin{array}{l}\text { Emergency } \\
\text { medicine: } 15 \text { MDs, } \\
346 \text { admissions }\end{array}$ & $\begin{array}{l}\text { All physicians } \\
(N=126) ; \text { all } \\
\text { admissions } \\
(N=9855)\end{array}$ & \\
\hline $\begin{array}{l}\text { Nicotine replacement } \\
\text { medication prescribing }\end{array}$ & $0.15(0.08,0.22)$ & $0.08(0.02,0.27)$ & $0.14(0.08,0.22)$ & 0.21 \\
\hline Quitline referral & $0.12(0.03,0.34)$ & $0.0(0.0,0.08)$ & $0.10(0.01,0.32)$ & 0.03 \\
\hline $\begin{array}{l}\text { Adding "tobacco use } \\
\text { disorder" to problem list }\end{array}$ & $0.25(0.13,0.46)$ & $0.21(0.04,0.60)$ & $0.25(0.12,0.47)$ & 0.54 \\
\hline \multirow{3}{*}{$\begin{array}{l}\text { Nicotine } \\
\text { replacement + quitline } \\
\text { referral + problem list } \\
\text { update } \\
\end{array}$} & $0.21(0.11,0.32)$ & $0.18(0.07,0.30)$ & $0.20(0.11,0.31)$ & 0.26 \\
\hline & \multicolumn{4}{|l|}{ Training level } \\
\hline & $\begin{array}{l}\text { Intern: } 44 \text { MDs, } \\
1906 \text { admissions }\end{array}$ & $\begin{array}{l}\text { Resident: } 60 \text { MDs, } \\
3322 \text { admissions }\end{array}$ & $\begin{array}{l}\text { Attending: } 22 \text { MDs, } \\
4627 \text { admissions }\end{array}$ & $P$ value \\
\hline $\begin{array}{l}\text { Nicotine replacement } \\
\text { medication prescribing }\end{array}$ & $0.14(0.08,0.21)$ & $0.16(0.08,0.24)$ & $0.10(0.07,0.22)$ & 0.40 \\
\hline Quitline referral & $0.11(0.02,0.34)$ & $0.07(0.01,0.19)$ & $0.32(0.07,0.58)$ & 0.004 \\
\hline $\begin{array}{l}\text { Adding "tobacco use } \\
\text { disorder" to problem list }\end{array}$ & $0.28(0.15,0.49)$ & $0.19(0.11,0.41)$ & $0.34(0.12,0.56)$ & 0.15 \\
\hline $\begin{array}{l}\text { Nicotine } \\
\text { replacement + quitline } \\
\text { referral + problem list } \\
\text { update }\end{array}$ & $0.23(0.11,0.32)$ & $0.15(0.11,0.29)$ & $0.27(0.09,0.43)$ & 0.17 \\
\hline
\end{tabular}


These correlation coefficients indicate a moderate-large effect size [12].

Because patient follow-up is ongoing and blinded, results addressing tobacco abstinence are not reported. Patient follow-up will be completed in the fall of 2016 .

\section{Discussion}

The E-STOPS prompt and order set resulted in a dramatic, sustained increase in the ordering of NRT, referrals to the state smokers' quitline and placement in patients' problem lists of tobacco use disorder. It resulted, as well, in near-universal notification of the patient's primary care provider that tobacco treatment had been initiated in the hospital. The treatment effect has persisted for the 2 years since its initiation in August, 2013, as shown in Table 2.

There was little variation in using E-STOPS components across physician specialty or level of training, with the exception of quitline referrals. It may be possible that the attending physicians, all hospitalists, were more familiar with quitlines than the residents and therefore more comfortable with making referrals. Or, perhaps because of their greater clinical experience, they recognize the impact of smoking on the range and severity of illness in hospitalized patients and are more willing to refer. Qualitative interviews currently in progress may elucidate these reasons further.

One potential limitation in our methods is that, during the consent process for providers, control physicians were asked to leave the room so that ESTOPS details could be reviewed with physicians randomized to the intervention. Thus, the intervention physicians had slightly greater exposure (perhaps 10$15 \mathrm{~min}$ ) with the study team, which may account for some of the response seen in the intervention. We could have spent additional time with the control physicians discussing non-tobacco items, as an attention control, but study resources did not permit this.

A key challenge in the dissemination and implementation of electronic health records has been to develop workflows that physicians and other providers find feasible and acceptable. A guiding principle of this project was to develop functionalities and workflows that physicians would find "minimally invasive." We aimed to provide inpatient physicians the ability to treat tobacco dependence with medication, counseling (via the quitline), and notification to primary care and other future providers that tobacco dependence treatment had begun.

Our data indicated that the E-STOPS seems to have had reasonable acceptability to the physician subjects, given its widespread use. To better understand the facilitators and barriers to using E-STOPS, we are currently conducting qualitative interviews with high and low utilizers.

As noted in the "Methods" section, we had to make critical decisions regarding prompt timing; triggers; choice of clinician specialty; and mapping clinicians to patients, order set functions, suppression logic, training, and audit/feedback mechanisms. These decisions may be handled differently by other medical centers, depending on local practices, workflows, and institutional culture. Of course, in routine clinical care, and outside the context of a clinical trial, physicians need not be randomized, thus allowing all admitted smokers to be treated by clinicians with access to E-STOPS.

Future work with E-STOPS may include implementation across the healthcare enterprise, so that smokers who present for care in any clinical venue-outpatient settings, emergency departments, inpatient units-may be treated. We are currently planning such a study in our medical center.

Critical to the program's implementation was gaining the support of key institutional leaders. These included the hospital's chief information officer, chief medical information officer, chief nursing officer, the director of the hospitalist service, and the directors of the residency programs in internal medicine (traditional, primary care, and medicine-pediatrics tracks) and emergency medicine. In addition, hospitalists and internal medicine chief residents were consulted during the design phase of E-STOPS, to ensure it was clinically feasible and compatible with current workflows. Importantly, in our health system, these key leaders embraced the study, highlighting the importance of improved tobacco treatment in clinical medicine.

\section{Conclusion}

Electronic health records can be configured to enhance the treatment of tobacco dependence among hospitalized smokers. Treatment modalities can be extended beyond the time of discharge. Electronic alerts and order sets can be implemented with reasonable uptake by physicians. The decision support tools are scalable across the Epic platform and likely to other EHRs. Whether this order set results in sustained tobacco abstinence is not yet known. Smoking cessation outcomes will be forthcoming in future reports.

Acknowledgements: We would like to thank the many hospitalists and residents in the Department of Medicine who helped us design and test the integrated tobacco order set. We also wish to thank our colleagues at Epic who assisted with E-STOPS design and implementation.

Authors' contributions: SLB designed and led the study and takes overall responsibility for its conduct. JR managed the study on a daily basis and contributed to intervention design. MD and ALH designed and tested the Electronic Support Tool and Orders for the Prevention of Smoking (E-STOPS). JD led the statistical analyses. JT, SS, and POC designed and led the training of the physicians. BT contributed to study design and endpoint selection. All authors contributed to drafting the manuscript.

Compliance with ethical standardsThe findings in this paper have not been previously published. The manuscript has not been submitted elsewhere. Partial results were reported at the 2014 and 2015 Annual Meetings of the Society for Research on Nicotine and Tobacco. The authors have full control of all primary data. We agree to allow the journal to review data, if requested. No animals were used in this research.

The study was approved by the Human Investigations Committee of Yale University. All subjects gave written informed consent. The study complies with the Declaration of Helsinki.

Funding: This study was supported by R18HL105208 from the National Heart, Lung, and Blood Institute of the National Institutes of Health.

Conflict of interest: The authors declare that they have no competing interests. 
Appendix

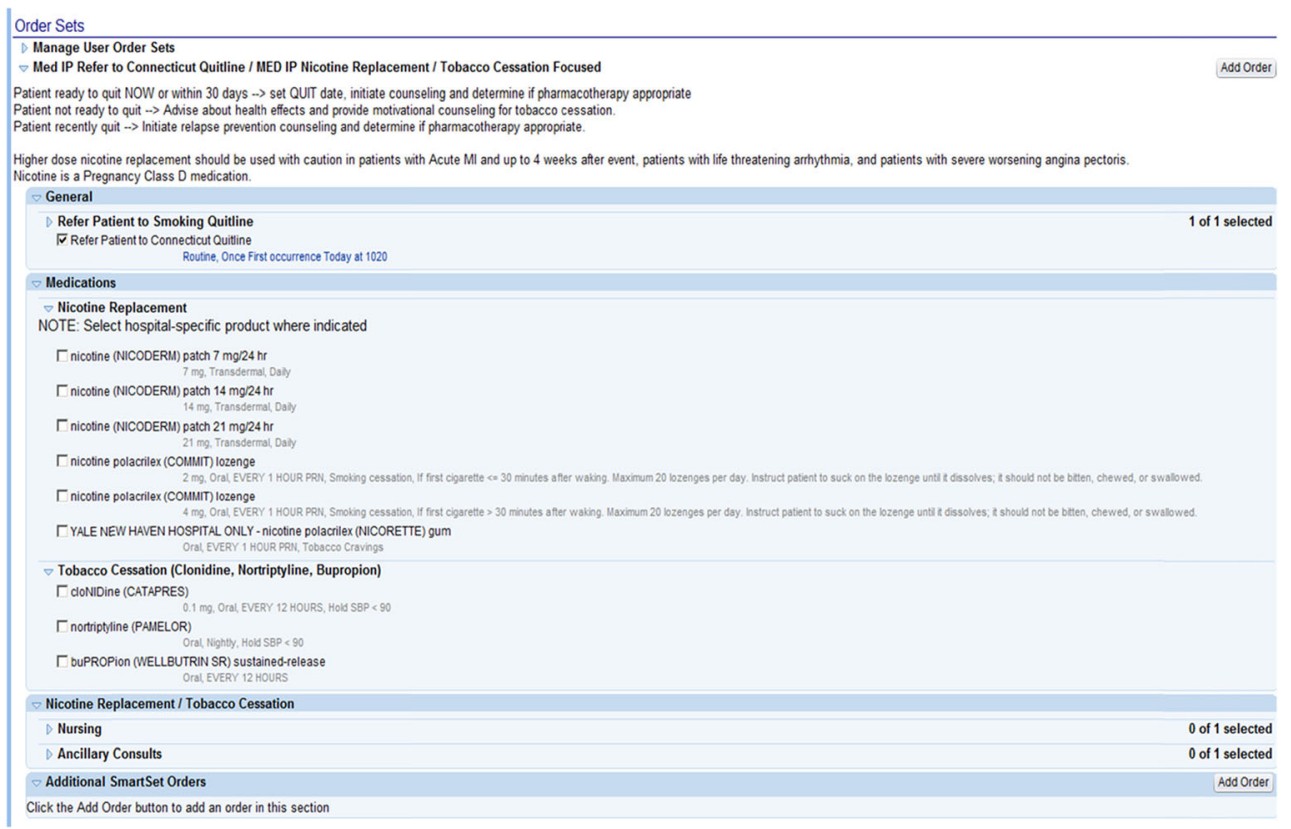

Fig. 3 | Tobacco order set

Patient's tobacco use addressed during hospitalization. Please consider continuing tobacco use treatment.

Varga, Susan, MD

Sent: Wed March 18, 2015 12:59 PM

To: Paek, Hyung M, MD

Fig. 4 | E-mail message to primary care physicians

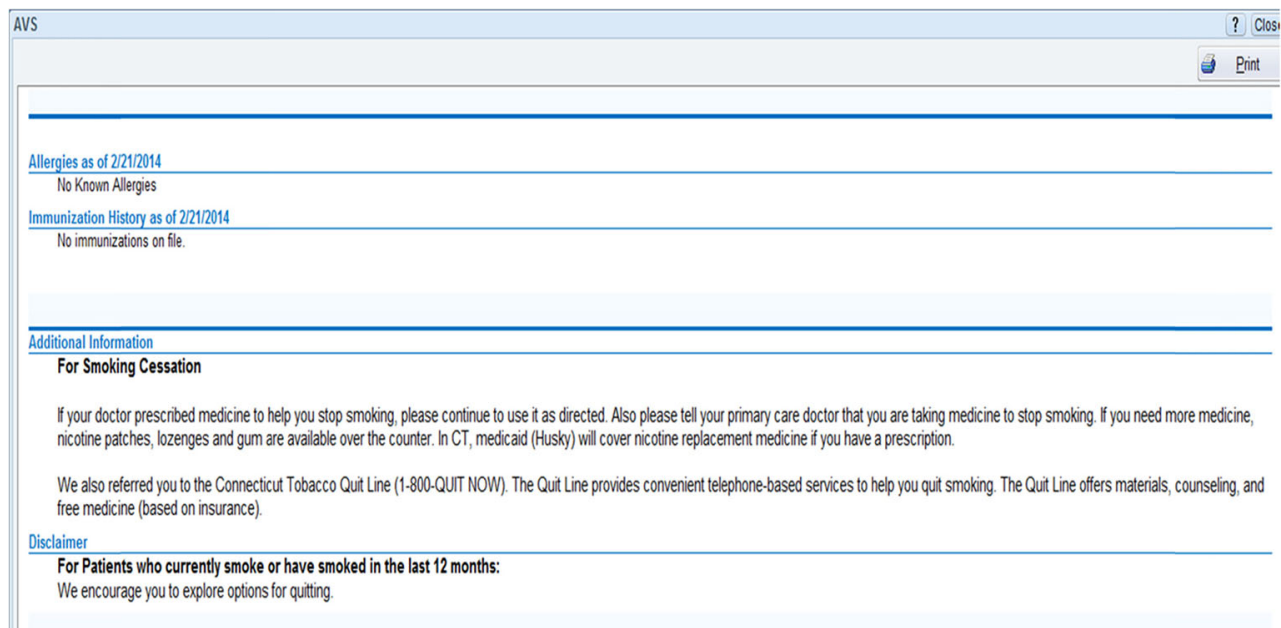


Dear Dr. Smith,

Thank you for participating in our study of decision support for smokers admitted to Yale- New Haven Hospital. The table below shows how you have used the order set, compared to the other physicians in the study, as of March 24, 2015.

\begin{tabular}{l|c|c|}
\multicolumn{1}{c}{} & \multicolumn{2}{c}{ BPA Actions Taken } \\
\cline { 2 - 3 } & You & Colleagues \\
\cline { 2 - 3 } Ordered NRT Medication & 4 & 3 \\
\cline { 2 - 3 } $\begin{array}{l}\text { Quitline Referrals } \\
\begin{array}{l}\text { InBasket Messages } \\
\text { sent to PC Physican }\end{array}\end{array}$ & 5 & 18 \\
\cline { 2 - 3 } $\begin{array}{l}\text { Tobacco Use Disorder } \\
\text { added to Problem List }\end{array}$ & 33 & 14 \\
\cline { 2 - 3 } & &
\end{tabular}

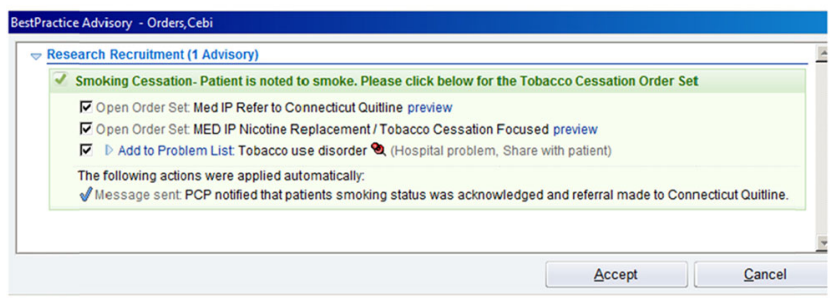

The screen shot shows you the BPA. Note that we pre-click for you a referral to the state Smoker's Quitline, and placement of 'Tobacco Use Disorder' in the patient's problem list.

If you have any questions about the study or the order set please contact me at steven bernstein@vale.edu or 203-737-3574.

Fig. 6 | Feedback report to physician subjects

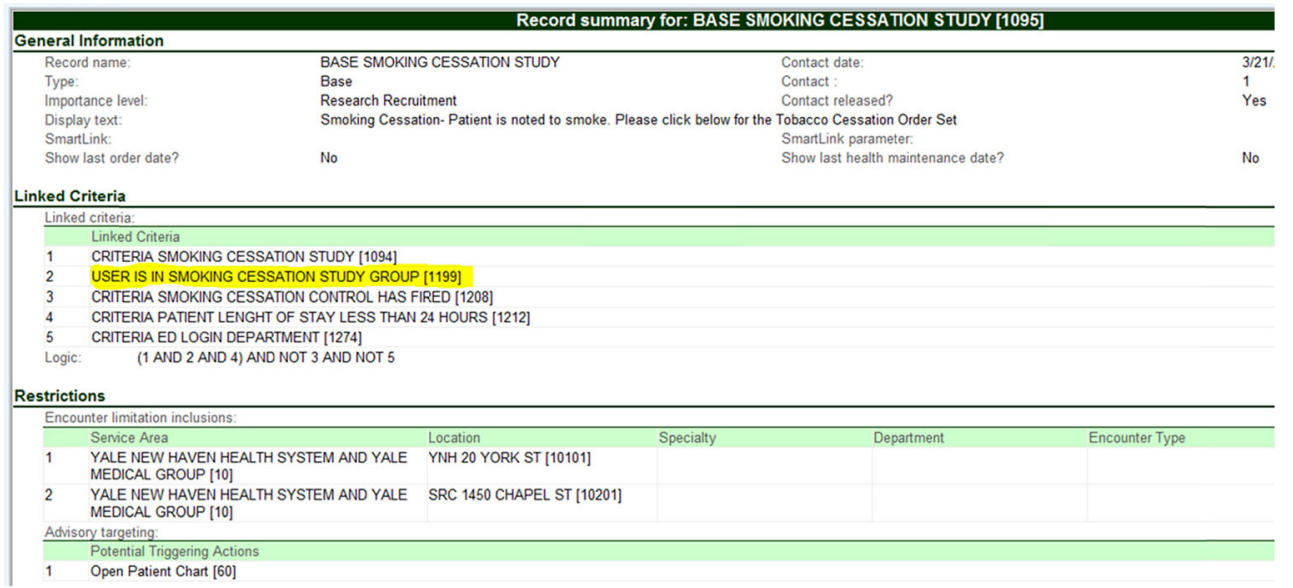

Fig. 7 | Best practice alert base record

1. Fiore, M. C., Goplerud, E., \& Schroeder, S. A. (2012). The Joint Commission's new tobacco-cessation measures-will hospitals do the right thing? $N$ Engl J Med, 366(13), 1172-1174. doi:10. 1056/NEJMp1115176.

2. Blumenthal, D., \& Tavenner, M. (2010). The meaningful use regulation for electronic health records. N Engl J Med, 363(6), 501504. doi:10.1056/NEJMp1006114.

3. Buntin, M. B., Burke, M. F., Hoaglin, M. C., \& Blumenthal, D. (2011). The benefits of health information technology: a review of the recent literature shows predominantly positive results. Health Aff, 30(3), 464-471. doi:10.1377/ hlthaff.2011.0178.
4. Jamal, A., McKenzie, K., \& Clark, M. J. (2009). The impact of health information technology on the quality of medical and health care: a systematic review. Health Information Management J, 38(3), 26-37.

5. Duffy, S. A., Karvonen-Gutierrez, C. A., Ewing, L. A., Smith, P. M., \& Team, V. T. T. (2010). Implementation of the tobacco tactics program in the Department of Veterans Affairs.J Gen Intern Med, 25, 3-10. doi:10.1007/s11606-009-1075-9.

6. Adsit, R., Fox, B., Tsiolis, T., Ogland, C., Simerson, M., Vind, L., \& Fiore, M. (2014). Using the electronic health record to connect primary care patients to evidence-based telephonic tobacco quitline services: a closed-loop demonstration 
project. Translational Behavioral Medicine, 1-9. doi:10.1007/ s13142-014-0259-y.

7. Rigotti, N. A., Munafo, M. R., \& Stead, L. F. (2008). Smoking cessation interventions for hospitalized smokers: a systematic review. Arch Intern Med, 168(18), 1950-1960. doi:10.1001/ archinte.168.18.1950.

8. Curran, G. M., Bauer, M., Mittman, B., Pyne, J. M., \& Stetler, C. (2012). Effectiveness-implementation hybrid designs: combining elements of clinical effectiveness and implementation research to enhance public health impact. Med Care, 50(3), 217-226. doi:10. 1097/MLR.0b013e3182408812.
9. Fiore, M. C., Jaén, C. R., Baker, T. B., Bailey, W. C., Benowitz, N. L., Curry, S. J., \& Wewers, M. E. (2008). Treating tobacco use and dependence: 2008 update. Rockville, MD: US Department of Health and Human Services.

10. Miller, W. R., \& Rollnick, S. (2002). Motivational interviewing: preparing people for change (2d ed.). New York: Guilford Press.

11. Mojica, W. A., Suttorp, M. J., Sherman, S. E., Morton, S. C., Roth, E. A., Maglione, M. A., \& Shekelle, P. G. (2004). Smoking-cessation interventions by type of provider: a meta-analysis. Am J Prev Med, 26(5), 391-401.

12. Cohen, J. (1988). Statistical power analysis for the behavioral sciences (2d ed.). New York: Lawrence Erlbaum Associates. 